Scientia Agricola

http://dx.doi.org/10.1590/0103-9016-2015-0399

\title{
Communication between agricultural scientists and statisticians: a broken bridge?
}

Marcin Kozak

University of Information Technology and Management in Rzeszów - Dept. Quantitative Methods in Economics, Sucharskiego 2 - 35-225 - Rzeszów.

Corresponding author <nyggus@gmail.com>

Edited by: Thomas Kumke

Received October 21, 2015

Accepted November 24, 2015

\begin{abstract}
Modern agricultural research requires a lot of statistics. Unfortunately, most up-todate statistical methods need advanced knowledge, often out of reach for agricultural researchers. Thus, efficient communication between researchers and statisticians is important for the development of agricultural knowledge. Many agricultural researchers claim that communication with statisticians is difficult. On the other hand, many statisticians claim that communication with agricultural researchers is not easy either. This being true, such poor communication can be a barrier to efficient agricultural research. The aim of this research is to study this phenomenon. Do agricultural researchers and statisticians see problems in their communication? What kinds of problems are they? I will try to answer these questions based on a study among scientists representing both groups.

Keywords: cooperation, expert study, statistics
\end{abstract}

\section{Introduction}

Contemporary agricultural research demands a lot of statistics (e.g., Schabenberger and Pierce, 2010). Sometimes it is rather simple, such as the t-test, correlation and regression analyses, and ANOVA for complete block designs together with pair-wise comparisons. Currently, such methods are often applied by non-statisticians. However, for several decades agricultural sciences have been calling for more and more advanced statistical methods. This - at least in part - is the effect of the dynamic development of statistics, especially after pairing applied statistics and computers (Efron, 1979). More and more complex experimental designs are used, and analyzing them can be difficult, too. As Nelsen (2002) says, "The basic statistical toolbox of the agricultural scientist was changed by the computer revolution and is continuing to be changed by advances in experimental designs, computer graphics, and measurement systems." Thus, whoever wishes to apply statistics in agriculture needs to have advanced statistical knowledge. Does it suffice to learn how to apply the t-test, correlation and regression analyses or ANOVA with that or other software? We all know it usually does not. Application of statistical procedures - even simple, at the basic level - can be tricky (e.g., Kozak, 2009). My experience as a statistical reviewer and editor for a number of journals shows that many simple mistakes are made in amazingly simple situations. This is quite often not because of poor knowledge of those who conducted the analyses, but because of their failure to recognize that the situation they deal with is actually not that simple. For example, we have analyzed various common mistakes made while using the correlation coefficient (Kozak et al., 2012). If a simple statistical method, such as the correlation coef-
Statistics have been a fundamental part of agricultural research, and statisticians can share in the success agriculture has had in keeping up with providing food to an ever growing population. Nelson (2002) ficient, can be applied incorrectly in numerous ways, we wonder what problems might arise from the use of more complex methods.

Statistical teaching has been a topic for deep studies, take for example, scientific journals such as Teaching Statistics, Journal of Statistics Education, Statistics Education Research Journal, or Technology Innovations in Statistics Education. Statistical teaching particularly to agricultural students has gained much less focus, however; for example the studies of López et al. (2009) or Onofri and Pannacci (2014) were published by the "Teaching corner" section of Communication in Biometry and Crop Science, whose aim is to publish papers concerned with teaching crop science and statistics to crop science. Statistical consultancy has not - at least in my opinion gained sufficient attention in the scientific literature either. Kravchuk and Rutley (2014) presented a project in statistical thinking and training to agricultural students who are later to become agricultural researchers. Piegorsch et al. (1998) stressed that efficient collaboration between environmental researchers and statisticians is crucial. Sprent (1970) discussed problems of statistical consultancy and he himself wrote: "It is often said of papers like this one that they merely state the obvious." Maybe this is why such topics are seldom discussed in scientific literature. It is a pity because papers like that of Sprent's and this one can help scientists understand that statistical consultancy is actually an art.

Efficient agricultural research requires complex statistics and complex statistics requires efficient communication between agricultural researchers and statisticians. Certainly, there are many examples of efficient and unproblematic collaboration between agricultural and statistical researchers. It does not mean, however, that such collaboration does not suffer from various 
problems. In the above mentioned paper, Sprent (1970) whose statistical consultancy dealt mainly with biology - provided a list of factors that can reduce the effectiveness of consultancy (Table 1). Easy to note is that most of the factors are on the statisticians' side, not on the agricultural researchers' side. A preliminary study I conducted among colleagues from both sides of the scene suggested that indeed various problems can occur.

This led me to conduct the present research, whose aim was to verify a research hypothesis that the communication between agricultural and statistical researchers needs to be improved. I approached this problem from the point of view of both sides: agricultural researchers and statisticians.

\section{Methods}

I emailed a number of agricultural researchers and statisticians working in agriculture and asked them simple questions concerned with cooperation between agricultural researchers and statisticians. Since my main agricultural domain is crop science, I focused on this field. I collected e-mail addresses from my own database, from crop science journals, as well as from departments throughout the world. I sent over 200 e-mails and received 38 responses. The experts were to choose from specialties statistician/agricultural researcher/both, and some of them did. Others, however, explained their specialties, based on which I decided whether such a person should be considered a statistician, agricultural researcher, or both. Of course, many statisticians working for agriculture have actually agricultural background (or even both backgrounds), which is why I decided to consider the three mentioned groups - and as it will occur soon, many statisticians claimed to be both statisticians and agricultural researchers.

I asked two questions:

QUESTION 1: "Have you ever personally encountered or heard of any problems in communication between statisticians and agricultural researchers?"
QUESTION 2: "If YES, please describe the kinds of problems they were."

My hope was that asking only these two questions would increase response rate. In addition, I thought that respondents would write more, seeing that there were just these two questions to answer.

Six researchers were included in a preliminary study in which these questions had slightly different meanings. However, since the differences were rather minor and the responses were interesting, I decided to include these responses in the regular study.

The analysis of the responses will be mainly qualitative in nature. I consider the respondents a non-representative sample of experts; thus, I will not pay too close attention to the frequency of responses. My most attention will go to the problems themselves as well as comments on them. Various experts pointed out several backgrounds and aspects of the problems. Their opinions helped me out to analyze the problems from various perspectives. However, the problems that have been pointed out by many experts will receive higher attention than those highlighted by one or two.

\section{Results}

Here is a summary of the experts who responded:

22 agricultural researchers

5 statisticians

10 both (most agricultural scientist with statistical experience)

1 other (a mechanical engineer, cooperating with both statisticians and agricultural scientists)

Twenty-five experts responded 'yes' to the first question, therefore, they have personally encountered or heard of problems in communication between statisticians and agricultural researchers; 13 experts responded 'no'. Nonetheless, some of those who responded 'no' actually provided a list of such problems. One of them even finished his response with the following sentence:

Table 1 - A list of factors that can reduce effectiveness of statistical consultancy offered by Sprent (1970). The items are quoted literally. Sprent indicated that the items are not ordered by importance.

1. The statistician may not have the time or background knowledge to become a full member of an experimental team.

2. The statistician may not have adequately adapted his outlook to the computer era, or may be by-passed by an experimenter with access to a statistical program package.

3. The statistician may be called in too late or dropped too early.

4. There is little or no training in the art of consultancy.

5. In some universities little account is given to the consultancy load in staffing and successful consultancy is not always a criterion for promotion.

6. In research institutes, there is a danger that over-specialization may lead to stagnation, as modern developments over a wide spectrum may be overlooked.

7. There is little informed criticism of consultancy and many of us are not keen on practicing the art of self-criticism. 
"Given these indicated problems, I should perhaps have answered YES..."

Below I present the analysis of the problems the respondents pointed out.

\section{Different languages and lack of experience in other fields}

(i) Statisticians fail to understand biology: Pure statisticians speak a language agricultural scientists may have problems to understand; pure statisticians are also inexperienced in the agricultural realm.

(ii) Agricultural researchers fail to understand statistics: Agricultural researchers speak a language statisticians may have problems to understand; agricultural researchers are inexperienced in the statistical realm.

As we see, this problem is split into two sub-problems that are concerned with both sides. In general, both groups tend to use technical vocabulary difficult to understand to the other side, this is especially true with theoretical (pure) statisticians. One respondent (BOTH) pointed out that shallow statistical knowledge of agricultural researchers limits proper understanding of the results of their experiments. A plant breeder claimed that lack of understanding of biological phenomena by statisticians may be problematic because they might have problems with suggesting a proper analysis of experimental data or modifying classical methods for untypical situations. The same respondent claimed that many statistical methods applied in life sciences are unknown by many statisticians. This same problem was raised also by the other agricultural scientists. It is crucial that statisticians understand the biological phenomena studied, but here it is the agricultural scientist's role. An agronomist claimed that communicating with a statistician is difficult if he or she "knows agricultural experiments only from the office and does not understand agricultural issues." Other agronomists mentioned that statisticians do not have to have deep knowledge of agriculture, but a more general knowledge.

Another agricultural researcher claimed that statisticians tend to speak in a strange, difficult-to-understand language. They are not willing to respond any questions and create the distance between them and the agricultural researcher. Yet, according to an applied agricultural statistician, statisticians often do not wish to learn biology while biologists do not wish to learn statistics.

Too late for a statistician to get involved (suggested by both statisticians and agricultural researchers)

Far too often, a statistician is asked to get involved in the research after conducting the experiment, at the stage of data analysis. It can be quite a problem. The statistician does not influence the experiment design, including the use of controls, number of replications, other design aspects and the method of recording observations. It often happens (when it is too late) that such designs are unacceptable and there is no way to improve them. As one of the respondents (BOTH) mentioned, agricultural researchers "often design studies that are not appropriate for the question or system that they are working with." While an incorrect data analysis can practically always be corrected, an incorrectly experiment design seldom can (it usually needs to be conducted once more, with a correct design). For some ill-designed experiments, no statistical method will help. Some experiments are even not randomized or replicated.

At the webpage of Statistical Service of the Agricultural Experiment Station of Utah State University (https://uaes.usu.edu/htm/uaes-support-services/statistical-consulting) we can find that:

"Faculty members and students involved in UAES research projects may use the services of the station's statistician to design experiments and analyze data. While the statistician may provide assistance at any stage of a project, the optimum time to engage her/his services is at the beginning when she/he can offer advice on experiment design and methods of gathering data that will ultimately improve projects by identifying and correcting potential problems."

Lack of precise aims of the study by agricultural researchers and, therefore, lack of precise expectations from statisticians (suggested by an agricultural scientist who after $\mathrm{PhD}$ re-qualified and became an applied statistician)

Quite often, statisticians are invited to collaborate with agricultural scientists; however, expectations are not provided. Instead, the statistician hears, "here are the collected data. Any ideas what to do with them?" It is an obvious misconception because a statistician at this stage of research should not be asked to offer research problems but to help resolve them through an appropriate analysis. This point is closely related to the previous one.

Agricultural researchers do not realize how complicated statistics is and how much it can do (suggested by a statistician)

One of the statisticians claimed, "Some other agricultural researchers may not realize that the subject of statistics is much larger and much more complicated than they think. In these cases, the statistician cannot live up to the expectations of the agricultural researchers." Interestingly, some agricultural researchers claimed that their experiments did not require advanced statistics. According to their opinion, basic knowledge gained in statistics courses at universities along with available statistical software suffice because what they normally need is ANOVA with F and LSD tests, correlation and regression analyses. Another agricultural scientist claimed that although she had not heard of problems in communication between the two groups, she thought that statistics was still underused in agricultural sciences. 
Lack of professional incentives for statistical consultancy (suggested by an agricultural researcher being a statistical consultant)

"Professional incentives are for pure statistical research, not consulting" (in words of a respondent who marked BOTH as his specialty). Quite a frequent problem is also that statisticians are treated as consultant personnel to help others, not as scientists. Thus, if one asks a statistician to help with his/her experiment, far too often it resembles asking a technician to take a sample or something like that. One forgets then that many statisticians are scientists too and can have different priorities. Of course, it depends on the position held by the statistician at the university, but if she/he holds a regular research position, consultancy is not their main duty.

Problems with fulfilling deadlines by statisticians (suggested by an agricultural researcher)

Most of the time statisticians do not have time to complete their assignments on time. They take on a specific assignment but fail to meet the deadline. It is most likely because they are overloaded with their own assignments. This makes this problem closely related to the previous one.

Limited statistical knowledge of statisticians (suggested by two agricultural researchers)

Many statistical methods applied in specific disciplines of life sciences, such as genetics, are unknown by many statisticians. Some statisticians who normally work with one kind of biological data have difficulties to grasp the character of another kind of biological data. An example was provided in which a statistician told geneticists that they had too few replicates $(3$ technical replicates for each of the 3 biological replicates, a standard in such molecular experiments). This statistician normally worked with multi-year field data, so, he criticized this number of replicates. The team (represented by one of the respondents) had to find another statistician who knew the specificity of molecular experiments.

The reason is specialization of statisticians, who are unable to learn all statistical methods in various agricultural disciplines. This actually is no problem but rather a misunderstanding, similar to claiming that an agricultural scientists should know all agricultural disciplines and specializations (say, a crop production specialists should know everything about crop genetics). One should not expect from statisticians knowledge of all statistical methods applied to all various agricultural problems. That being said, it is worth noting that many statisticians who have no agricultural or more generally biological knowledge attempt to work with agricultural data without gaining sufficient knowledge.

Different statisticians, different methods (suggested by an agricultural researcher)

One of the agricultural scientists mentioned that different statisticians advise different methods to use for one dataset or to verify one hypothesis. It indeed can be a problem and I have myself encountered such problems more than once. If one thinks more about it, this problem is serious: given a data set and a set of questions, employing different methods or approaches to data analysis can lead to different inferences, interpretations and conclusions. And we know that for a particular dataset, there are so many various possibilities. One can choose among different approaches, methods, models, estimation techniques, significance levels, and so many other things. Still, two persons can differently interpret the same results, for example, by stressing different aspects of the results. It is not a strange thing, actually. As an editor for various journals, I have encountered numerous situations in which (i) authors and reviewer(s) disagreed in terms of their statistical analysis; (ii) reviewers offered completely different opinions on statistical analysis in a manuscript they reviewed; (iii) editor(s) and reviewer(s) disagreed in terms of their statistical analysis; and (iv) take whatever combinations of editors (there can be more than one for one manuscript), reviewers and authors, and construct the disagreement party. When analyzing the same dataset with colleagues, I myself - this time as a statistician - disagreed with my colleagues on which method to choose, etc. Although it is a normal thing in science that should not come as a surprise, it is something that does not make statistics and statisticians popular among agricultural scientists.

The community knows nothing. Why should I? (suggested by an agricultural researcher)

The agricultural community has poor statistical knowledge. If so, should one lose one's time to learn statistics? What sense does it make since the community will not be able to evaluate one's knowledge and will not appreciate it? On the one hand, it is a narrow-minded thought that misses the point. On the other hand, young adepts learn from their masters. When they see that statistics is not appreciated, will they spare their time on learning and using it, risking to be criticized by their supervisors and the whole community? My guess is that this problem is not too common these days although a decade or so it might have been. Still, it was pointed out by one of the experts as an important problem.

Overuse of "youngsters" (suggested by both statisticians and agricultural researchers)

An agricultural researcher pointed out that statisticians in the group she consults with are students at the statistics service on campus. These students lack a good understanding of biology and, therefore, cooperation with them is far-too-often just a waste of time.

A statistician pointed out that "we are often consulting with students and young researchers, and the senior people do not attend those who have designed the experiments." Nonetheless, another statistician (who 
indicated being BOTH a statistician and an agricultural researcher) highlighted that "About half of the consulting that I do is for researchers who have already been to a statistical consulting lab and have already consulted with someone, usually a graduate student. I routinely find that these graduate students know very little about experimental designs, especially complex and unusual designs." Thus, we see that not only are young researchers overused as statistical consultants, but their supervisors (agricultural researchers) also use their students as a channel of communication with statisticians. I myself have encountered such problems at least several times. Once a professor in agricultural sciences sent a $\mathrm{PhD}$ student to consult with me. It was about an experiment this student had not even seen, but, the professor was sure it would suffice to send this student to offer the consultancy. The problem was that the experiment was not even randomized! Therefore, I actually had to give this student the very basic lesson of randomization, though I was aware that it was his professor who should be listening to the lesson.

On the webpage of Statistical Service of the Agricultural Experiment Station of Utah State University (https://uaes.usu.edu/htm/uaes-support-services/statistical-consulting) it is stressed that, "It is very important that major professors accompany their graduate students to at least the initial meeting so everyone involved has an opportunity to communicate clearly about the project."

Incorrect literature (suggested by a researcher who indicated being both a statistician and an agricultural researcher)

As I already mentioned, statisticians themselves have various views on particular statistical methods, and, in the very same situations, some of them will choose a different path of analysis than others. According to one of the experts, erroneous papers on statistical methodology "prompt agricultural researchers to use inferior analyses, which results in less insight and slower progress." Therefore, especially in the early stages of their development, some methodologies can be incorrect or misleading or simply need to be developed further. But, of course, such under-developed methods are used, which adds to the literature with the incorrect use of the method. Such papers are then a source of methodological solutions and further applications. Let us note that it is a normal situation in science development that incorrect methodologies are proposed, used, and mirrored in later applications. Sometimes it is difficult to decide which method is better and even the best statisticians will differ in their opinions. The Fisher-Neyman argument on hypothesis testing is one such example.

\section{"Lucky me!"}

Some of the agricultural researchers mentioned that they did not hear of the problems with communication with statisticians. Some others did hear about such problem but they themselves had not encountered these problems because they were lucky to efficiently cooperate with a statistician. Sometimes it was an experienced statistician, sometimes a student. An ideal situation, then, is when an agricultural researcher is inclined to cooperate with a statistician who is equally inclined to cooperate with this agricultural researcher and both of them have sufficient knowledge in their disciplines along with basic background in the other discipline.

In short, some researchers are lucky to find colleagues with whom they are able to cooperate efficiently while others are not. This can be for various reasons, sometimes on their side, other times on the other side.

\section{Particular methodological problems raised by the respondents}

Here is a list of problems concerned with particular topics raised by the respondents. I will not dig into them for the simple reason that this is outside the main scope of this paper. In particular, these topics are not actual problems regarding communication between agricultural researchers and statisticians, but they can be topics for which such communication should be efficient. Here is the list:

- GGE biplots and many papers with erroneous methodological elements.

- Stability indices, which are often used incorrectly.

- Interpretation of usual quantities produced during data analysis, and particularly the interpretation of confidence intervals and significance levels.

- Fixed vs random effects.

- Non-replicated trials in organic farming.

- Distinction between standard error of a mean and that of a difference.

- Lack of understanding about replication scales, how to define experimental units, and restrictions that arise from confounding treatments with experimental units.

- Modern reductionism associated with mixed models.

- Number of replications (especially when a statistician is not accustomed to working with a particular topic in which a small number of replications is a standard).

- Combining many trials in one statistical analysis.

- Dealing with year in field trials.

- Randomization and replication.

I do not list these topics just for the record. They have been indicated as problematic by those agricultural researchers and statisticians who work directly with them. Clearly some of topics can be a bone of contention. Thus, agricultural statisticians should keep these topics in mind while working on the statistical methodology for agricultural sciences. 


\section{Discussion}

The community of agricultural scientists and statisticians working with agriculture recognizes that communication between these two groups is not perfect. A list of problems that were raised by agricultural researchers and statisticians is too long to be treated lightly. Some of the problems themselves can be serious. Based on the opinion of the experts from agricultural researchers and statisticians, we can construct a list of recommendations that might help improve this communication.

Statisticians should be more sympathetic to agricultural researchers; however, agricultural researchers should be equally more sympathetic to statisticians. Statisticians should not ignore the agricultural knowledge; nevertheless, agricultural researchers should not equally ignore the statistical knowledge. Statisticians should avoid technical language, as should agricultural researchers.

Statisticians should be aware that their knowledge can be limited; however, agricultural researchers should not think that there is just one "statistics" and that every single statistician should know everything about statistics, otherwise being just an ignoramus. Statistics is science, statistics is art of science (Agresti and Franklin, 2013); statisticians - the scientists and the artists - are not workers in a factory, and as such, they will often choose different colors and textures of their works. This does not have to be a drawback of statistics. It does not have to, though sometime it is.

Statisticians must not be involved when it is too late, rather, they should be consulted at practically every stage of research. Otherwise, their job will mostly be to cure a wrong experiment rather than to help answer research questions by means of proper experimentation and data analysis.

Importance of statistical consultancy is difficult to overestimate in agricultural research (e.g., Kravchuk and Rutley, 2014). Thus, statistical consultancy should never be treated too superficially by both, statisticians and agricultural researchers, sending their students to represent them at consultancy.

It is time to get back to Sprent's observations, made 45 years ago (Sprent, 1970; refer to Table 1). Our results reinforce some of them. First of all, a statistician's background knowledge is important. Second, the statistician must not be involved too late or dropped too early. Consultancy is lightly weighted and is seldom a criterion for promotion for statisticians. Statisticians are "not keen on practicing the art of self-criticism", which of course makes communication with agricultural researchers difficult. I would, however, claim that statisticians are not a different species from other scientists, and other scientists are not keen on practicing the art of self-criticism either. Unfortunately, Sprent's (1970) claim that there is little or no training in the art of consultancy still holds.

Reassuring is that some agricultural researchers reported to have been lucky to find a good statistical col- laborator. Such stories prove that efficient collaboration between the two groups of scientists is possible and does happen. On the other hand, others were not so lucky and had to struggle with problems in such communication, which often led to problems with statistical analysis of their data.

I do not wish to claim here that statistics is a remedy to everything and that agricultural research needs a lot of complex and advanced statistics. On the contrary, statistics should be used only when there is such a need, and under particular circumstances, simple statistics can be much better than complex one. Scientific literature is full of controversy on the overuse of statistics in science and much of this controversy is true (e.g., Siegfried, 2010). It only confirms that correct statistics is important - by "correct" I mean correct indeed, not overly complex and difficult.

The final conclusion is that agricultural researchers should keep looking for consultants among statisticians until they find one with whom cooperation will be efficient and pleasant. Such a statistician will be one who can understand (to a certain extent) which biological phenomena the statistical analysis is to deal with; who has sufficient background in statistics for biology; who will not be biased against some agricultural disciplines for being accustomed to other agricultural (or not) disciplines; and who will be aware of his or her limited knowledge. However, agricultural researchers also need to be good collaborators. The good agricultural collaborator is someone who has sufficient knowledge of the biological phenomena one is going to work with; who has basic background in statistics for biology, enough to understand what the statistical colleague is going to address; and who will be aware of one's limited knowledge.

\section{Acknowledgments}

I wish to thank all the following researchers who responded to my request and took part in this study: Ricardo Azevedo, Bhoja Raj Basnet, Zoltan Bedo, Jan Bocianowski, Michael Casler, Douglas Cook, Adam Davis, Muhammad Farooq, Johannes Forkman, Dariusz Gozdowski, Hugh G. Gauch, Zofia Nizioł-Łukaszewska, Manjit Kang, Wojtek Krzanowski, Phillip Miklas, Andrea Onofri, Jan Paderewski, Hans-Peter Piepho, Emanuele Radicetti, Kulasekaran Ramesh, Saeed Rauf, Paulo C. Rodrigues, Victor Sadras, Stanisław Samborski, Dorota Sienkiewicz-Paderewska, Ahmed Sharif, Darshan Sharma, Rolf Sommer, Eva Stoltz, Sivakumar Sukumaran, Dana Sullivan, Ana M. Tarquis, Tihana Teklić, Edzard van Santen, Tom Warkentin, Christie Williams, and the two others who preferred to remain anonymous.

\section{References}

Agresti, A.; Franklin, C. 2013. Statistics: The Art and Science of Learning from Data. 3ed. Pearson, London, UK. 
Efron, B. 1979. Computers and the theory of statistics: thinking the unthinkable. Siam Review 21: 460-480.

Kozak, M. 2009. Analyzing one-way experiments: a piece of cake of a pain in the neck? Scientia Agricola 66: 556-562.

Kozak, M.; Krzanowski, W.; Tartanus, M. 2012. Use of the correlation coefficient in agricultural sciences: problems, pitfalls and how to deal with them. Anais da Academia Brasileira de Ciências 84: 1147-1156.

Kravchuk, O.; Rutley, D.L. 2014. Engaging entry level researchers in agriculture in statistical communication and collaboration: why? and how? p.345-364. In: Topics from Australian conferences on teaching statistics. Springer, New York, NY, USA.

López, M.V.; Fabrizio, M.C.; Plencovich, M.C. 2009. Evaluation of design and variability concepts among students of agriculture. p. 173-180. In: Bidgood, P.; Hunt, N.; Jolliffe, I., eds. Assessment methods in statistical education: an international perspective (VISA Project). Wiley, New York, NY, USA.
Nelsen, T.C. 2002. The state of statistics in agricultural science. Journal of Agricultural, Biological, and Environmental Statistics 7: 313-319.

Onofri, A.; Pannacci, E. 2014. Spreadsheet tools for biometry classes in crop science programmes. Communications in Biometry and Crop Science 9: 43-53.

Piegorsch, W.W.; Smith, E.P.; Edwards, D.; Smith, R.L. 1998. Statistical advances in environmental science. Statistical Science 13: 186-208.

Schabenberger, O.; Pierce, F.J. 2010. Contemporary statistical models for the plant and soil sciences. CRC Press, Boca Raton, FL, USA.

Siegfried, T. 2010. Odds are, it's wrong: science fails to face the shortcomings of statistics. Science News 177: 26-29.

Sprent, P. 1970. Some problems of statistical consultancy. Journal of the Royal Statistical Society. Series A 133: 139-165. 